\title{
Wharton's Jelly Derived Mesenchymal Stem Cells: Future of Regenerative Medicine? Recent Findings and Clinical Significance
}

\author{
Ilona Kalaszczynska ${ }^{1,2}$ and Katarzyna Ferdyn ${ }^{3}$ \\ ${ }^{1}$ Department of Histology and Embryology, Center for Biostructure Research, Medical University of Warsaw, \\ Chalubinskiego 5, 02-004 Warsaw, Poland \\ ${ }^{2}$ Centre for Preclinical Research and Technology, Banacha 1b, 02-097 Warsaw, Poland \\ ${ }^{3}$ Polish Stem Cell Bank, Grzybowska 2/41, 00-131 Warsaw, Poland \\ Correspondence should be addressed to Ilona Kalaszczynska; ikalaszczynska@wum.edu.pl
}

Received 12 December 2014; Accepted 2 March 2015

Academic Editor: Josep Maria Pujal

Copyright (C) 2015 I. Kalaszczynska and K. Ferdyn. This is an open access article distributed under the Creative Commons Attribution License, which permits unrestricted use, distribution, and reproduction in any medium, provided the original work is properly cited.

Around 5 million annual births in EU and 131 million worldwide give a unique opportunity to collect lifesaving Wharton's jelly derived mesenchymal stem cells (WJ-MSC). Evidences that these cells possess therapeutic properties are constantly accumulating. Collection of WJ-MSC is done at the time of delivery and it is easy and devoid of side effects associated with collection of adult stem cells from bone marrow or adipose tissue. Likewise, their rate of proliferation, immune privileged status, lack of ethical concerns, nontumorigenic properties make them ideal for both autologous and allogeneic use in regenerative medicine applications. This review provides an outline of the recent findings related to WJ-MSC therapeutic effects and possible advantage they possess over MSC from other sources. Results of first clinical trials conducted to treat immune disorders are highlighted.

\section{Introduction}

Interest in mesenchymal stem cells has been kindled in 1960s as the result of Friedenstein's observations who reported that the bone marrow stroma can generate bone [1]. It was later shown that bone marrow stromal cells have chondrogenic and adipogenic properties and a high ability for selfrenewal [2]. Even though there is debate on the technical name (mesenchymal or multipotent stem cells), there is an agreement to the acronym "MSC". Since their original description, presence of MSC has been proven in many adult and embryonic tissues such as adipose tissue [3], muscle [4], peripheral blood [5], lung [6], heart [7], corneal stroma [8], dental pulp [9], placenta [10], endometrium [11], amniotic membrane [12], and Wharton's jelly [13]. MSC have the capability to differentiate into wide range of specialized cells of mesodermal origin: bone cells, cartilage, fat, cardiomyocytes, muscle fibers, renal tubular cells, and break germ layer commitment and differentiate into cells of ectodermal origin, for example, neurons, and endodermal origin, such as hepatocytes and pancreatic islets cells. Due to the above properties, MSC are considered as a new emerging treatment option and therapeutic agent in regenerative medicine. MSC therapeutic potential can be executed by direct replacement of injured tissue cells or by paracrine effect on surrounding environment, indirectly supporting revascularisation, protecting tissue from stress-induced apoptosis, and appropriately modulating inflammatory reaction. Results of MSCbased cell therapies are very promising in various clinical fields, based on in vitro and in vivo research results and more than 400 clinical trials registered.

\section{Are All MSC Phenotypically and Functionally Equivalent? Age Does Matter}

The lifelong perseverance of adult MSC in the body makes them particularly susceptible to the accumulation of cellular 
damage, which can lead to cell death, senescence, or loss of regenerative function and in extreme cases to neoplastic transformation. In contrast, neonatal MSC such as Wharton's jelly derived MSC, in their short, prenatal life are spared from proaging factors. Decreased repair capacity and increased susceptibility to degenerative diseases may stem from the fact that the function of stem cells declines with age. There is increasing evidence that the age of the donor tissue affects several properties of mesenchymal stem cells [1416]. By means of single cell transcriptional analysis, it was shown that aged adipose tissue derived MSC (ADSC) are significantly compromised in their ability to support the vascular network formation and are unable to rescue ageassociated impairments in cutaneous wound healing [17]. Further, bone marrow derived MSC have lesser myogenic potential and engraftment properties than developmentally early MSC [18]. As recently shown, one of the mechanisms implicated in MSC aging involves Akt/mTOR pathway and its inhibition prevents the development of age-related phenotype and maintains MSC morphology, self-renewal, and differentiation capacity [19]. Further studies demonstrate that the expression levels of inflammatory response genes change with age and that the age-dependent decrease in expression of several cytokine and chemokine receptors is important for the migration and activation of BMSC. By adoptive transfer of aged BMSC to young endotoxemic mice, authors showed that aged cells lacked the anti-inflammatory, protective effect of their young counterparts, which indicate that BMSC undergo an age-related decline in their immunomodulatory activity [20].

A growing body of evidence suggests that elevated activity of certain proteins can have beneficial effects on aging and aging-related diseases. Among them is SITR1, NAD+-dependent protein deacetylase, which is downregulated in rodent bone marrow derived MSC with aging [21] or human MSC with increasing passages [22]. It is also shown that expression of genes related to senescence such as CHEK1, p16 ink4a increases in ADSC with age where at the same time proapoptotic regulators levels, ATR, TNF $\alpha$, and $\mathrm{NF} \kappa \mathrm{B}$ decreased [23]. Aging alters the availability of $\mathrm{CD} 45^{-} / \mathrm{CD} 34^{+} / \mathrm{CD} 133^{+} \mathrm{ADSC}$ and their angiogenic properties [24].

Healthspan of mesenchymal stem cells also depends on maintaining physiological level of reactive oxygen species (ROS). However, during lifetime and exposure to environmental stress, ROS levels can increase dramatically. This may result in significant damage to cell structures and promote MSC aging. In support of the hypotheses, increased levels of ROS have been reported in aging BMSC [14]. Furthermore, exposure of adult ADSC isolated from old rat donors to $\mathrm{H}_{2} \mathrm{O}_{2}$ resulted in decreased expression of integrin and reduced phosphorylation of focal adhesion kinase Src and FAK. In consequence, intramyocardial transplantation of aged ADSC into acute myocardial infarction model rats resulted in a decreased survival rate of old MSC in the infarct region. The authors conclude that the old ADSC are more sensitive to the microenvironmental ROS and their therapeutic effectiveness is impaired [25]. In another study, in a rat myocardial infarction model, authors evaluated regenerative capacity of human MSC derived from young versus older patients (1-5 versus 50-70 years old). "Young" MSC outperformed "older" MSC in cardiac parameters: ejection fraction, fractional shortening, and left ventricular end-diastolic and end-systolic volumes. Increase in vascular density and decrease in metalloproteinases levels and activity were observed in recipients of "young" BMSC [26]. Similarly, MSC obtained from young individuals have been induced to undergo neuroectodermal differentiation in vitro, but this effect could not be reproduced in BMSC from elderly individuals [27]. This proves adult BMSC unsuitable for successful cell replacement strategies for neurologic diseases in elderly patients in autologous setting.

During normal aging cells divide and telomeres that are essential to maintain the stability of genomes shorten. Even though MSC in their niche are relatively quiescent, adult MSC during their lifespan undergo significantly more divisions shortening their telomeres than neonatal cells. Thus, in comparison to MSC from adult tissues, WJ-MSC at such an early embryonic state retain telomere at highest possible length, which protects them from premature loss of viability.

A very important issue, which does not apply to Wharton's jelly derived MSC, is an exposure of adult MSC during the lifetime to intrinsic (e.g., inflammatory mediators) and extrinsic factors, for example, nonsteroidal antiinflammatory drugs (NSAIDs) commonly used in patients to treat inflammation, pain, and fever. These factors may greatly inflect MSC viability or plasticity. Effects of NSAIDs on the MSC potential for proliferation and differentiation towards the osteogenic and chondrogenic lineages were investigated [28]. It was shown that type $\mathrm{X}$ collagen, a marker of late stage chondrocyte hypertrophy, is constitutively expressed by mesenchymal stem cells (MSC) from osteoarthritis patients treated with NSAID, Naproxen [29]. Similarly, osteogenic differentiation of MSC was affected, and downregulation of mineral deposition in the extracellular matrix was observed [30]. These results contradicted previous findings, demonstrating no effect of several types of NSAIDs on osteogenic differentiation. However, in vitro chondrogenesis, shown by glycosaminoglycans production, was significantly inhibited. These findings suggest that NSAIDs may inhibit MSC chondrogenic differentiation and disrupt endochondral bone formation [31]. Despite discrepancies, it is evident that NSAID can alter certain essential processes involved in the MSC performance as therapeutic agent.

The therapeutic potential of adult MSC can be also affected by donors lifestyle. Although high-fat diet induced type 2 diabetes did not affect the number of cells per gram of adipose tissue, analysis of differentiation potential of ADSC derived from high-fat diets fed mice showed a higher adipogenic potential and a lower endothelial differentiation potential in vitro compared to control group [32]. Impaired response to osteogenic stimuli was also shown for ADSC from obese patients. Ranx2 expression was 6-9 times lower than in control cells and mineralization nodules were fewer and smaller [33]. Altered properties of ADSC and BMSC were also demonstrated by others. Surprisingly, in obese mice, increased frequency of BMSC and subcutaneous ADSC was shown. However, adipogenic, osteogenic, and chondrogenic 
potential of BMSC from obese mice was diminished. ADSC showed increased adipogenic and osteogenic differentiation but decreased CD105 expression consistent with inefficient chondrogenic potential [34]. Observed phenotype might be associated with increased levels of free fatty acids (FFA) in plasma of obese patients. Consistent with this notion, palmitate (most abundant FFA in plasma of obese patients) treated BMSC showed induced expressions of adipogenic transcription factors, namely, CCAAT enhancer-binding protein, $\mathrm{C} / \mathrm{EBP} \beta, \mathrm{C} / \mathrm{EBP} \alpha$, and $\mathrm{PPAR} \gamma$, and in consequence increased adipogenic differentiation [35]. The elevated level of FFA in obese individuals may initiate events leading to irreversible changes in MSC from bone marrow and adipose tissue. Consistently, another study confirmed upregulation of adipocyte lineage commitment genes, such as Tcf 21, Pitx2, and Lif. At the same time, the expression of "stemness" genes (Sdf1, Tbx15) was downregulated [36].

Obesity is one of the factors increasing the risk of developing type 2 diabetes [37]. Metabolic diseases such as diabetes may influence stem cell niche and endogenous MSC properties. Therefore feasibility of autologous stem cell therapy in diabetic patients may not be possible or at least significantly hampered. Indeed, it was shown that BMSC from diabetic patients, although phenotypically similar to healthy human BMSC, expressed insulin, C-peptide, and other pancreatic markers not observed in control healthy cells [38]. Furthermore, in a recent study, investigators demonstrated that diabetes alters ADSC milieu and diminishes the cells' ability to establish a vascular network both in vitro and in vivo in wound healing mouse model [39]. It could be expected, since significant decrease of major angiogenic genes (Vegf- $a, F g f-2$, and Pdfg-a) and their associated receptors ( $C x c r-4, F g f r-2$, and $P d g f r-a)$ expression was observed.

Collectively, this observation indicates that the microenvironment in disease influences the stem cells. Therefore, tissues from patients with various metabolic diseases may not be satisfactory as an autologous source of mesenchymal stem cells for therapeutic purposes [40].

According to WHO statistics, 35\% of adults aged 20 and over are overweight and $11 \%$ are obese (as of 2008), while $8 \%$ are living with diabetes. Taking into account the fact that the passing of time and changes in MSC microenvironment due to disease translate into reduced effectiveness of tissue regeneration, MSC derived from Wharton's jelly offer a good clinical alternative to adult MSC. In the near future, autologous use of these cells will be possible due to growing interest in Wharton's jelly banking.

\section{More Differences between Adult and Wharton's Jelly Derived MSC}

The superiority of WJ-MSC is based not only on adult MSC limitations but on its own prominent capacity.

5 million annual births in EU and 131 million worldwide give a unique opportunity to collect umbilical cord (UC), isolate lifesaving mesenchymal stem cells, and cryopreserve them for allogeneic or autologous application as soon as the need arises. The unlimited availability of tissue source is not the only advantage of WJ-MSC.

3.1. Isolation Efficiency: Number Does Matter. Most of clinical applications of MSC require a large number of cells for transplantation. Therefore, abundance, easiness of isolation, and proliferative potential may be deciding factors while choosing a source of MSC. The amount of mesenchymal stem cells, which can be obtained from bone marrow, is very limiting. Only 0.001 to $0.01 \%$ of mononuclear cells were reported [41], while $1 \mathrm{~g}$ of adipose tissue yields approximately $5 \times 10^{3}$ stem cells, which is 500 -fold greater than in the bone marrow [42]. The isolation efficiency from Wharton's jelly is high and ranges from 1 to $5 \times 10^{4}$ cells $/ \mathrm{cm}$ of umbilical cord [43]. Side-by-side comparison of MSC from bone marrow adipose tissue and Wharton's jelly demonstrated that WJMSC have highest proliferative capacity among tested cell types [44]. MSC from the umbilical cord can be isolated either by enzymatic digestion or by explant culture of 1-3 mm pieces of the UC [45-48]. However, at p0 explant culture method yielded 2.8 times more cells per gram of UC than enzymatic digestion [46]. Of great importance for large-scale MSC production is the fact that population doubling time of WJ-MSC isolated by enzymatic method is significantly longer [49]. Furthermore, enzymatic digestion may induce cellular damage, as MSC isolated by explant method demonstrated increased viability. Another advantage of explant method is growth factors release from tissue pieces during in vitro culture. Large amounts of different growth factors were reported in Wharton's jelly $[46,50]$. Among them, bFGF is noteworthy, as it regulates self-renewal and positively affects osteogenic and chondrogenic differentiation of MSC while added to the growth medium [51-54]. Wharton's jelly released bFGF mediates stimulation of WJ-MSC growth in a way external supplementation provides.

To further increase isolation and culture efficiency, several modifications of explant culture methods and dedicated devices were proposed $[55,56]$. Interestingly, a device designed for repeated explant culture at the same time prevented floating of Wharton's jelly pieces [55]. By sequential transfer of device with fragments of tissue strung on the steel rings, investigators reported 15-20 times higher number of cells derived by this method. However, mentioned method seems to be labor-intensive, especially for fast and largescale production of WJ-MSC. Another approach, proposed by others in order to optimize method of MSC isolation, is based on isolation of WJ-MSC from large pieces or the entire cord piece [56-58]. The only concern posed by this method is possible heterogeneity of derived cells. However, no differences in cell surface antigen expression, population doubling time, or pattern of adipogenic, osteogenic, and chondrogenic differentiation were observed [56]. Therefore, explant culture methods of Wharton's jelly only or entire umbilical cord are worth of consideration for labor-, time-, and cost-effective WJ-MSC isolation for clinical purposes.

3.2. Properties of WJ-MSC Crucial for Clinical Application. Phenotypic analysis performed by many groups proved that 
WJ-MSC fit the minimal criteria outlined for MSC by the International Society for Cellular Therapy [59]. WJMSC express mesenchymal markers such as CD73, CD90, and CD105 and are negative for endothelial, CD31, and hematopoietic, CD45, CD34, markers [13, 60, 61]. What sets WJ-MSC apart and makes them more unique and useful for therapeutic applications from adult MSC is their more primitive characteristics [62]. It is already well known that WJ-MSC display several features of embryonic stem cells (ESC), especially regarding the expression of ESC-like stem cell markers and wide spectrum of differentiation beyond mesodermal origin. Expression of pluripotency genes, Oct4, Nanog, and SOX-2, was reported for WJ-MSC [46, 63, 64], although much lower than in ESC [65]. Modest expression of pluripotency genes might explain why WJ-MSC are not tumorigenic (do not form teratomas) as demonstrated in numerous preclinical studies in immunocompetent and immunodeficient animals $[66,67]$. Furthermore, as recently shown by the comprehensive analysis of WJ-MSC and ESC transcriptome, the high expression level of several tumor suppressor genes may explain the lack of in vivo teratoma induction [65]. The same mechanism might be one of many responsible for attenuation of tumor growth by WJ-MSC. Moreover, large amounts of various cytokines and growth factors are secreted by WJ-MSC which result in cancer cells in vitro and tumor in vivo growth inhibition. WJMSC cell lysates or conditioned medium inhibited growth of breast adenocarcinoma, ovarian carcinoma, osteosarcoma [68], benign neoplastic keloid cells [69], bladder tumor [70], or lymphoma cells [71] in vitro. Similarly, intratumorally administered cell lysates and WJ-MSC conditioned medium inhibited mammary carcinoma, osteosarcoma, and pancreatic and lung tumor growth and resulted in decreased tumor sizes and weights in vivo [72-75]. The antitumor effect of WJ-MSC was shown to be accomplished through multiple mechanisms. Antiproliferative properties of WJ-MSC were demonstrated by cell counting, MTT, BrdU or $\left[{ }^{3} \mathrm{H}\right]-$ thymidine incorporation assays, cell cycle regulators, and flow cytometric analysis. In lung or bladder tumor cells, cell cycle progression was blocked in G0/G1 phase and resulted in the downregulation of cyclin A2 and its associated kinase, cdk2, downregulation of Akt, and upregulation of tumor suppressor p53 phosphorylation, as well as cyclin dependent kinases inhibitor, p21 protein level [70, 73, 76]. In the breast cancer cells, synthesis of DNA was inhibited and arrest of cells in G2 phase of the cell cycle observed [74]. By cleaved caspase $3 / 9$ upregulation in cancer cells, WJ-MSC were executing its proapoptotic effect $[70,77]$. Consistently, increase in tumor cell death driven by WJ-MSC was due to an inhibitory effect on cancer "survival genes," such as Bcl-2, Bcl-xL, Survivin, Mcl-1, and cIAP-1 [78]. Autophagy was also indicated as one of the mechanisms responsible for anticancer effect of WJMSC. Upregulation of autophagy-related BAX, ATG5, ATG7, and BECLIN-1 genes was observed in osteosarcoma [68] and keloid cells [69] upon treatment with WJ-MSC conditioned medium or lysates.

Still, we must undertake far-reaching precautions and moderate enthusiasm in the implementation of WJ-MSC as anticancer therapy, since reports of tumor supporting function were recently published in regard to esophageal carcinoma [79] and renal cancer [80].

3.3. Immunoprivileged Status of WJ-MSC. The ability to modulate immunological responses ranks WJ-MSC as an important compatible stem cell type for therapeutic applications in allogeneic setting. The mechanisms of immunoprivilege are still investigated; however, low MHC-I level and absence of MHC-II expression protect them from NK-mediated lysis $[81,82]$. Despite the fact that they synthesize, though low, amounts of MHC class I, WJ-MSC do not demonstrate immunogenicity. It can be attributed to the lack of costimulatory molecules-CD 40, CD80, CD86 expression, and high levels of inhibitors of immune response: indoleamine2,3-dioxygenase (IDO) and prostaglandin E2 (PGE2). Of particular importance is the fact that WJ-MSC express high levels of leukocyte antigen G6 (HLA-G6), the same which is produced by trophoblast and protects the embryo from immune-based destruction [83]. Notably, in such a nonchallenging to allogeneic immune cells setup, immunorejection of WJ-MSC seems not to pose a threat and HLA matching may not be required before MSC transplantation. Therefore, administration of immunosuppressive drugs is not required, thereby protecting the patient against their side effects. Besides mechanisms described above, immunoprivilege of WJ-MSC depends on immunosuppressive functions mediated by the wealth of paracrine factors as well as cell-cell contact (reviewed in detail in Jyothi Prasanna and Jahnavi [84] and Ma et al. [85]).

The question remains if immunoprivilege of allogeneic WJ-MSC upon differentiation is maintained. Although use of allogeneic MSC in clinic is considered safe, reports of limited survival and long-term engraftment of MSC in such a setting are published. For instance, increased immunogenicity of BMCS was shown upon endothelial and myogenic differentiation [86]. A shift in the expression of immune antigens MHC-I and MHC-II made BMSC susceptible to immune rejection in a rat model of myocardial infarction. In another case, when the composite of hydroxyapatite and allogeneic BMSC was implanted, none of the allografts survived or showed osteogenic differentiation. Treatment with FK506 immunosuppressant prevented rejection and stimulated allogeneic BMSC osteogenic differentiation in vivo [87]. So far, such discouraging results were not reported for WJ-MSC. Results published so far demonstrated that the chondrogenic differentiation of human WJ-MSC did not change the level of expression of the aforementioned genes except for a very minor increase in the level of MHC class I. Costimulatory factors were not expressed and could not activate $\mathrm{T}$ lymphocytes. Moreover, high levels of potent inhibitors of immune response (IDO, HLA-G, and PGE2) were detected in differentiated WJ-MSC [88]. In vivo analysis of pig WJ-MSC injected into damaged rat brain revealed successful engraftment, proliferation, and differentiation into tyrosine hydroxylase positive neuronal cells without requiring immune suppression [89]. So far, it seems that state of immunoprivilege is stable in WJ-MSC upon multidirectional differentiation. Further studies are 
required in order to prove sustained immunoprivilege status of WJ-MSC upon differentiation which may depend on the species or stimulating factor.

\section{Clinical Applications of WJ-MSC}

The first clinical trial to test the feasibility and efficacy of WJMSC therapy was registered in 2008. By November 2014, the public clinical trials database http://www.clinicaltrials.gov/ has shown 51 clinical trials using WJ-MSC for a very wide range of therapeutic applications (Table 1, keywords used: Wharton's jelly mesenchymal stem cells or umbilical cord mesenchymal stem cells). Most of these trials are safety studies (Phase I) and proof of concept (Phase II) with very few in Phase III (comparison of a new treatment to the standard treatment).

To date, the results of studies listed are not published yet. However, rapidly increasing interest in WJ-MSC clinical application has resulted already in several published observations.

4.1. Type 1 Diabetes Mellitus. In a double blind study 15 patients with newly onset type 1 diabetes mellitus received 2 doses of $1.5-3.2 \times 10^{7}$ of WJ-MSC at 4-week interval by intravenous delivery [90]. Strikingly, within a period of 24 months, in 3/15 patients insulin supplementation was discontinued and in $8 / 15$ and $3 / 15$ the daily dosage was reduced by more than $50 \%$ and $15-50 \%$, respectively. Only 1 patient did not benefit from WJ-MSC treatment. In the control group, not subjected to WJ-MSC treatment, the dose of insulin increased gradually. No adverse reactions, chronic side effects were reported during the follow-up study.

4.2. Type 2 Diabetes Mellitus. In a nonplacebo controlled study, 22 patients (17 on insulin therapy) received WJ-MSC [91]. A first dose of $10^{6} / \mathrm{kg}$ was infused intravenously. Five days later, another dose was delivered to the pancreas via the splenic artery. Within 6 months after treatment, from 17 patients receiving insulin, 7 became insulin free and 5 had a reduction in insulin requirement by $\geq 50 \%$, in the rest $\leq 50 \%$, with only 1 patient who did not respond to MSC therapy. Interestingly, WJ-MSC treatment resulted in a significant decrease in proinflammatory IL- $1 \beta$ and IL-6 plasma level. This may have in vivo implications because IL-6 is an osteoclastogenic stimulus. Therefore, treatment of diabetic patients may also protect them from osteoporosis. Such effect may not be achieved by bone marrow derived MSC from aged patients, since BMSC from a mouse model of early aging secrete higher levels of IL-6 and have higher osteoclastogenesis-inducing activity [92]. Moreover, adult aged BMSC cocultured with activated T-cells were found to secrete more IL-6 than younger cells [93].

In both studies, parameters such as levels of glycated hemoglobin, C-peptide, and fasting plasma glucose were monitored. All parameters improved, HbAlc level gradually decreased, and progressive increase of C-peptide and Cpeptide/glucose ratio was observed.
4.3. Systemic Lupus Erythematosus (SLE). SLE is common and potentially fatal autoimmune disease resulting in renal, neural, cardiovascular, musculoskeletal, or cutaneous injury. In a nonplacebo controlled study, 40 patients received 2 doses of $10^{6} / \mathrm{kg}$ of WJ-MSC at 1-week interval by intravenous delivery [94]. No transplantation related side effects were observed. During 12 months of follow-up study 13/40 and $11 / 40$ achieved major or partial clinical response manifested by significant improvement in renal function, decrease in SLEDAI (Systemic Lupus Erythematosus Disease Activity Index) and BILAG (British Isles Lupus Assessment Group) scoring. 16/40 patients did not respond to MSC therapy. At 9 months after treatment, 7 patients experienced disease relapse; therefore, the authors concluded that repeated infusion with WJ-MSC is necessary to avoid disease relapse.

4.4. Late-Onset Hemorrhagic Cystitis (HC). HC is a common complication after allogeneic hematopoietic stem cell transplantation, characterized by hemorrhagic inflammation of the bladder. Late-onset of $\mathrm{HC}$ is frequently associated with ongoing graft-versus-host disease (GVHD). Seven patients received $1-3$ doses of $0.8-1.6 \times 10^{6} / \mathrm{kg}$ WJ-MSC by injection through a central line. As a result of stem cell treatment, gross hematuria dramatically resolved in 2-12 days, while the time to remission for patients not treated with WJ-MSC was significantly longer [95].

Reported results confirm that WJ-MSC are viable option as an adjuvant treatment for late-onset hemorrhagic cystitis.

The above results of pioneering studies demonstrated the effectiveness of WJ-MSC infusion for immune disorders.

\section{Conclusions}

Taken together, the clinical implication of oxidative stress, telomere length, DNA damage and disease is impaired therapeutic potential of MSC isolated from aged patients. These changes in MSC biology indicate that aged patients may require an alternative source of stem cells for treatment. The high efficiency of WJ-MSC recovery, the minimal ethical concerns associated with its acquirement and use, low immunogenicity, and the fact that they are from healthy, young donors make them an ideal source of MSC for autologous and allogeneic applications. Private and public banking of perinatal tissues gains popularity. During MSC preparation for clinical applications, observance of national and international regulations regarding standards and procedures is required. Quality management systems already in place in functioning tissue/cell banks guarantee high standards for the donation, procurement, testing, processing, storage, and ptdistribution of the WJ-MSC. Therefore, as the off-the-shelf product, WJ-MSC can be applied safely, immediately, and on demand. The next several years should abound in results of clinical applications of WJ-MSC and hopefully prove their invaluable properties. 


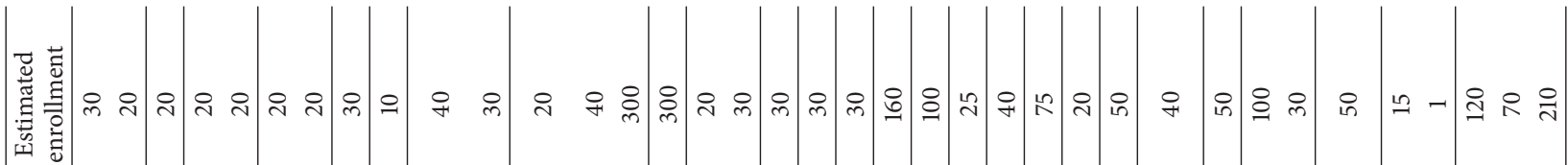

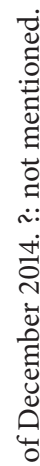

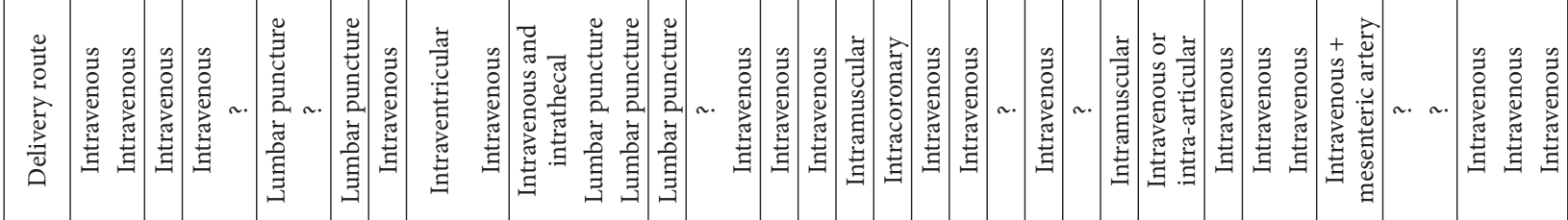

ก

宫
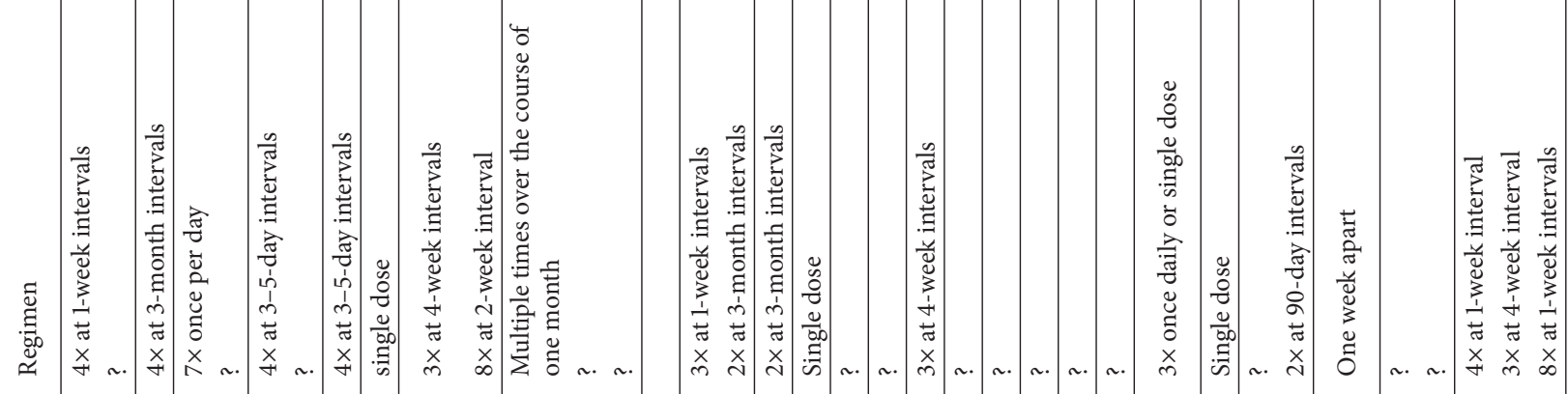

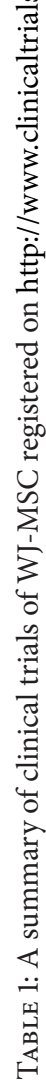

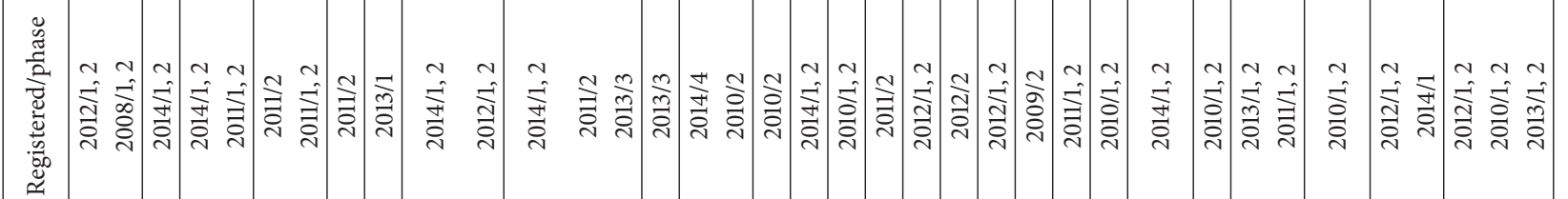

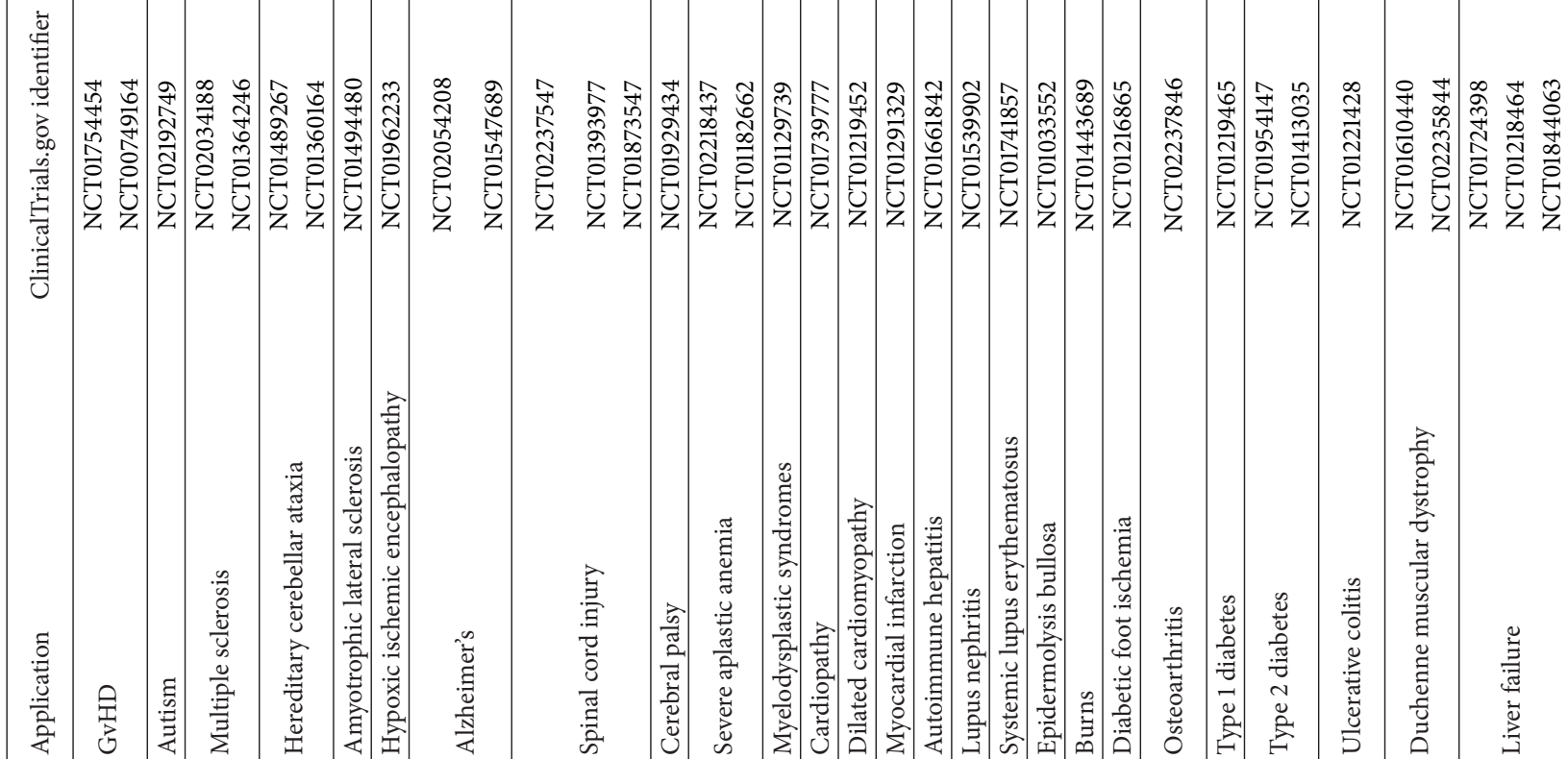




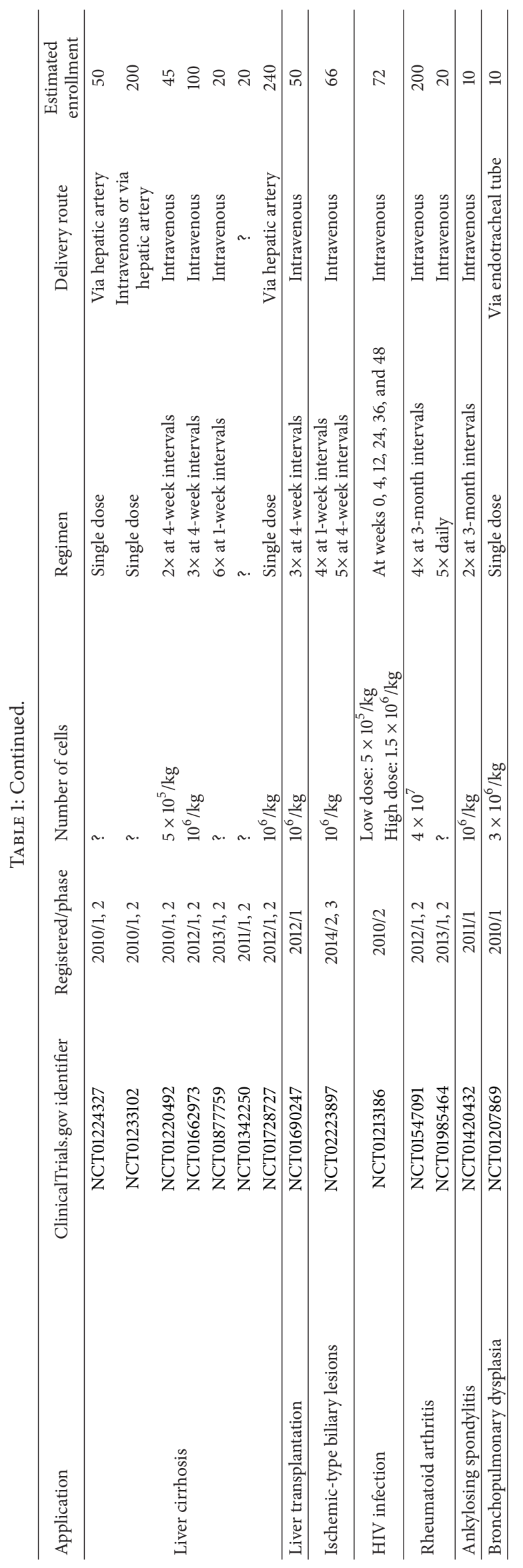




\section{Conflict of Interests}

The authors declare that there is no conflict of interests regarding the publication of this paper.

\section{References}

[1] A. J. Friedenstein, I. I. Piatetzky-Shapiro, and K. V. Petrakova, "Osteogenesis in transplants of bone marrow cells," Journal of Embryology and Experimental Morphology, vol. 16, no. 3, pp. 381-390, 1966.

[2] M. Owen, "Marrow stromal stem cells," Journal of Cell Science, no. 10, pp. 63-76, 1988.

[3] P. A. Zuk, M. Zhu, H. Mizuno et al., "Multilineage cells from human adipose tissue: implications for cell-based therapies," Tissue Engineering, vol. 7, no. 2, pp. 211-228, 2001.

[4] J. T. Williams IV, S. S. Southerland, J. Souza, A. F. Calcutt, and R. G. Cartledge, "Cells isolated from adult human skeletal muscle capable of differentiating into multiple mesodermal phenotypes," American Surgeon, vol. 65, no. 1, pp. 22-26, 1999.

[5] I. Kassis, L. Zangi, R. Rivkin et al., "Isolation of mesenchymal stem cells from G-CSF-mobilized human peripheral blood using fibrin microbeads," Bone Marrow Transplantation, vol. 37, no. 10, pp. 967-976, 2006.

[6] K. T. Hennrick, A. G. Keeton, S. Nanua et al., "Lung cells from neonates show a mesenchymal stem cell phenotype," The American Journal of Respiratory and Critical Care Medicine, vol. 175, no. 11, pp. 1158-1164, 2007.

[7] J. J. H. Chong, V. Chandrakanthan, M. Xaymardan et al., "Adult cardiac-resident MSC-like stem cells with a proepicardial origin," Cell Stem Cell, vol. 9, no. 6, pp. 527-540, 2011.

[8] M. J. Branch, K. Hashmani, P. Dhillon, D. R. E. Jones, H. S. Dua, and A. Hopkinson, "Mesenchymal stem cells in the human corneal limbal stroma," Investigative Ophthalmology and Visual Science, vol. 53, no. 9, pp. 5109-5116, 2012.

[9] S. Gronthos, M. Mankani, J. Brahim, P. G. Robey, and S. Shi, "Postnatal human dental pulp stem cells (DPSCs) in vitro and in vivo," Proceedings of the National Academy of Sciences of the United States of America, vol. 97, no. 25, pp. 13625-13630, 2000.

[10] Y. Fukuchi, H. Nakajima, D. Sugiyama, I. Hirose, T. Kitamura, and K. Tsuji, "Human placenta-derived cells have mesenchymal stem/progenitor cell potential," Stem Cells, vol. 22, no. 5, pp. 649-658, 2004.

[11] N. H. Cho, Y. K. Park, Y. T. Kim, H. Yang, and S. K. Kim, "Lifetime expression of stem cell markers in the uterine endometrium," Fertility and Sterility, vol. 81, no. 2, pp. 403-407, 2004.

[12] F. Alviano, V. Fossati, C. Marchionni et al., “Term amniotic membrane is a high throughput source for multipotent mesenchymal stem cells with the ability to differentiate into endothelial cells in vitro," BMC Developmental Biology, vol. 7, article 11, 2007.

[13] H. S. Wang, S. C. Hung, S. T. Peng et al., "Mesenchymal stem cells in the Wharton's jelly of the human umbilical cord," Stem Cells, vol. 22, no. 7, pp. 1330-1337, 2004.

[14] A. Stolzing, E. Jones, D. McGonagle, and A. Scutt, "Age-related changes in human bone marrow-derived mesenchymal stem cells: consequences for cell therapies," Mechanisms of Ageing and Development, vol. 129, no. 3, pp. 163-173, 2008.
[15] K. Huang, D.-H. Zhou, S.-L. Huang, and S.-H. Liang, "Agerelated biological characteristics of human bone marrow mesenchymal stem cells from different age donors," Zhongguo Shi Yan Xue Ye Xue Za Zhi, vol. 13, no. 6, pp. 1049-1053, 2005.

[16] K. Stenderup, J. Justesen, C. Clausen, and M. Kassem, "Aging is associated with decreased maximal life span and accelerated senescence of bone marrow stromal cells," Bone, vol. 33, no. 6, pp. 919-926, 2003.

[17] D. Duscher, R. C. Rennert, M. Januszyk et al., "Aging disrupts cell subpopulation dynamics and diminishes the function of mesenchymal stem cells," Scientific Reports, vol. 4, article 7144, 2014.

[18] C.-H. Ting, P.-J. Ho, and B. L. Yen, "Age-related decreases of serum-response factor levels in human mesenchymal stem cells are involved in skeletal muscle differentiation and engraftment capacity," Stem Cells and Development, vol. 23, no. 11, pp. 12061216, 2014.

[19] B. Gharibi, S. Farzadi, M. Ghuman, and F. J. Hughes, "Inhibition of Akt/mTOR attenuates age-related changes in mesenchymal stem cells," STEM CELLS, vol. 32, no. 8, pp. 2256-2266, 2014.

[20] M. L. Bustos, L. Huleihel, M. G. Kapetanaki et al., "Aging mesenchymal stem cells fail to protect because of impaired migration and antiinflammatory response," The American Journal of Respiratory and Critical Care Medicine, vol. 189, no. 7, pp. 787-798, 2014.

[21] H. Chen, X. Liu, W. Zhu et al., "SIRT1 ameliorates age-related senescence of mesenchymal stem cells via modulating telomere shelterin," Frontiers in Aging Neuroscience, vol. 6, article 103, 2014.

[22] H.-F. Yuan, C. Zhai, X.-L. Yan et al., "SIRT1 is required for long-term growth of human mesenchymal stem cells," Journal of Molecular Medicine, vol. 90, no. 4, pp. 389-400, 2012.

[23] E. U. Alt, C. Senst, S. N. Murthy et al., "Aging alters tissue resident mesenchymal stem cell properties," Stem Cell Research, vol. 8, no. 2, pp. 215-225, 2012.

[24] R. Madonna, F. V. Renna, C. Cellini et al., "Age-dependent impairment of number and angiogenic potential of adipose tissue-derived progenitor cells," European Journal of Clinical Investigation, vol. 41, no. 2, pp. 126-133, 2011.

[25] L. Li, Y. Guo, H. Zhai et al., "Aging increases the susceptivity of MSCs to reactive oxygen species and impairs their therapeutic potency for myocardial infarction," PLoS ONE, vol. 9, no. 11, Article ID e111850, 2014.

[26] M. Fan, W. Chen, W. Liu et al., "The effect of age on the efficacy of human mesenchymal stem cell transplantation after a myocardial infarction," Rejuvenation Research, vol. 13, no. 4, pp. 429-438, 2010.

[27] A. Hermann, C. List, H.-J. Habisch et al., "Age-dependent neuroectodermal differentiation capacity of human mesenchymal stromal cells: limitations for autologous cell replacement strategies," Cytotherapy, vol. 12, no. 1, pp. 17-30, 2010.

[28] M. Müller, O. Raabe, K. Addicks, S. Wenisch, and S. Arnhold, "Effects of non-steroidal anti-inflammatory drugs on proliferation, differentiation and migration in equine mesenchymal stem cells," Cell Biology International, vol. 35, no. 3, pp. 235-248, 2011.

[29] A. Almaawi, H. T. Wang, O. Ciobanu et al., "Effect of acetaminophen and nonsteroidal anti-inflammatory drugs on gene expression of mesenchymal stem cells," Tissue Engineering-Part A, vol. 19, no. 7-8, pp. 1039-1046, 2013.

[30] O. Salem, H. Wang, A. M. Alaseem et al., "Naproxen affects osteogenesis of human mesenchymal stem cells via regulation 
of Indian hedgehog signaling molecules," Arthritis Research \& Therapy, vol. 16, no. 4, article R152, 2014.

[31] I. Pountos, P. V. Giannoudis, E. Jones et al., "NSAIDS inhibit in vitro MSC chondrogenesis but not osteogenesis: implications for mechanism of bone formation inhibition in man," Journal of Cellular and Molecular Medicine, vol. 15, no. 3, pp. 525-534, 2011.

[32] V. Lamontagne, S. E. Akoum, I. Cloutier, and J.-F. Tanguay, "High-fat diets-induced metabolic alterations alter the differentiation potential of adipose tissue-derived stem cells," Open Journal of Endocrine and Metabolic Diseases, vol. 3, no. 3, pp. 197-207, 2013.

[33] M. Roldan, M. Macias-Gonzalez, R. Garcia, F. J. Tinahones, and M. Martin, "Obesity short-circuits stemness gene network in human adipose multipotent stem cells," The FASEB Journal, vol. 25, no. 12, pp. 4111-4126, 2011.

[34] C.-L. Wu, B. O. Diekman, D. Jain, and F. Guilak, "Diet-induced obesity alters the differentiation potential of stem cells isolated from bone marrow, adipose tissue and infrapatellar fat pad: the effects of free fatty acids," International Journal of Obesity, vol. 37, no. 8, pp. 1079-1087, 2013.

[35] E. Y. Park, C. E. Yeum, G. Seo, J.-Y. Lee, S.-B. Lee, and G.T. Chae, "The adipogenic effect of palmitate in mouse bone marrow-derived mesenchymal stem cells," Tissue Engineering and Regenerative Medicine, vol. 10, no. 2, pp. 77-85, 2013.

[36] B. Oñate, G. Vilahur, S. Camino-López et al., "Stem cells isolated from adipose tissue of obese patients show changes in their transcriptomic profile that indicate loss in stemcellness and increased commitment to an adipocyte-like phenotype," $B M C$ Genomics, vol. 14, article 625, 2013.

[37] M. A. Lazar, "How obesity causes diabetes: not a tall tale," Science, vol. 307, no. 5708, pp. 373-375, 2005.

[38] S. M. Phadnis, S. M. Ghaskadbi, A. A. Hardikar, and R. R. Bhonde, "Mesenchymal stem cells derived from bone marrow of diabetic patients portrait unique markers influenced by the diabetic microenvironment," Review of Diabetic Studies, vol. 6, no. 4, pp. 260-270, 2009.

[39] R. C. Rennert, M. Sorkin, M. Januszyk et al., "Diabetes impairs the angiogenic potential of adipose-derived stem cells by selectively depleting cellular subpopulations," Stem Cell Research \& Therapy, vol. 5, no. 3, article 79, 2014.

[40] Z. Kočí, K. Turnovcová, M. Dubský et al., "Characterization of human adipose tissue-derived stromal cells isolated from diabetic patient's distal limbs with critical ischemia," Cell Biochemistry and Function, vol. 32, no. 7, pp. 597-604, 2014.

[41] M. F. Pittenger, A. M. Mackay, S. C. Beck et al., "Multilineage potential of adult human mesenchymal stem cells," Science, vol. 284, no. 5411, pp. 143-147, 1999.

[42] J. K. Fraser, I. Wulur, Z. Alfonso, and M. H. Hedrick, "Fat tissue: an underappreciated source of stem cells for biotechnology," Trends in Biotechnology, vol. 24, no. 4, pp. 150-154, 2006.

[43] M. L. Weiss, S. Medicetty, A. R. Bledsoe et al., "Human umbilical cord matrix stem cells: preliminary characterization and effect of transplantation in a rodent model of Parkinson's disease," Stem Cells, vol. 24, no. 3, pp. 781-792, 2006.

[44] P. R. Amable, M. V. T. Teixeira, R. B. V. Carias, J. M. Granjeiro, and R. Borojevic, "Protein synthesis and secretion in human mesenchymal cells derived from bone marrow, adipose tissue and Wharton's jelly,' Stem Cell Research and Therapy, vol. 5, no. 2, article 53, 2014.

[45] P. Salehinejad, N. B. Alitheen, A. M. Ali et al., "Comparison of different methods for the isolation of mesenchymal stem cells from human umbilical cord Wharton's jelly," In Vitro Cellular \& Developmental Biology: Animal, vol. 48, no. 2, pp. 75-83, 2012.

[46] J. H. Yoon, E. Y. Roh, S. Shin et al., "Comparison of explantderived and enzymatic digestion-derived MSCs and the growth factors from Wharton's jelly," BioMed Research International, vol. 2013, Article ID 428726, 8 pages, 2013.

[47] S. Karahuseyinoglu, O. Cinar, E. Kilic et al., "Biology of stem cells in human umbilical cord stroma: in situ and in vitro surveys," Stem Cells, vol. 25, no. 2, pp. 319-331, 2007.

[48] K. Seshareddy, D. Troyer, and M. L. Weiss, "Method to isolate mesenchymal-like cells from Wharton's Jelly of umbilical cord," Methods in Cell Biology, vol. 86, pp. 101-119, 2008.

[49] Y.-F. Han, R. Tao, T.-J. Sun, J.-K. Chai, G. Xu, and J. Liu, "Optimization of human umbilical cord mesenchymal stem cell isolation and culture methods," Cytotechnology, vol. 65, no. 5, pp. 819-827, 2013.

[50] K. Sobolewski, A. Małkowski, E. Bańkowski, and S. Jaworski, "Wharton's jelly as a reservoir of peptide growth factors," Placenta, vol. 26, no. 10, pp. 747-752, 2005.

[51] S. Tsutsumi, A. Shimazu, K. Miyazaki et al., "Retention of multilineage differentiation potential of mesenchymal cells during proliferation in response to FGF," Biochemical and Biophysical Research Communications, vol. 288, no. 2, pp. 413-419, 2001.

[52] F. Ng, S. Boucher, S. Koh et al., "PDGF, TGF-beta, and FGF signaling is important for differentiation and growth of mesenchymal stem cells (MSCs): transcriptional profiling can identify markers and signaling pathways important in differentiation of MSCs into adipogenic, chondrogenic, and osteogenic lineages," Blood, vol. 112, no. 2, pp. 295-307, 2008.

[53] L. A. Solchaga, K. Penick, J. D. Porter, V. M. Goldberg, A. I. Caplan, and J. F. Welter, "FGF-2 enhances the mitotic and chondrogenic potentials of human adult bone marrow-derived mesenchymal stem cells," Journal of Cellular Physiology, vol. 203, no. 2, pp. 398-409, 2005.

[54] L. A. Solchaga, J. J. Auletta, E. A. Zale, and J. F. Welter, "Fibroblast growth factor-2 enhances expansion of human bone marrow-derived mesenchymal stromal cells without diminishing their immunosuppressive potential," Stem Cells International, Article ID 235176, 10 pages, 2011.

[55] Z. Chang, T. Hou, J. Xing et al., "Umbilical cord wharton's jelly repeated culture system: a new device and method for obtaining abundant mesenchymal stem cells for bone tissue engineering," PLoS ONE, vol. 9, no. 10, Article ID el10764, 2014.

[56] F. Hendijani, H. Sadeghi-Aliabadi, and S. Haghjooy Javanmard, "Comparison of human mesenchymal stem cells isolated by explant culture method from entire umbilical cord and Wharton's jelly matrix," Cell and Tissue Banking, vol. 15, no. 4, pp. 555565, 2014.

[57] G. La Rocca, R. Anzalone, S. Corrao et al., "Isolation and characterization of Oct-4+/HLA-G+ mesenchymal stem cells from human umbilical cord matrix: differentiation potential and detection of new markers," Histochemistry and Cell Biology, vol. 131, no. 2, pp. 267-282, 2009.

[58] C. De Bruyn, M. Najar, G. Raicevic et al., "A rapid, simple, and reproducible method for the isolation of mesenchymal stromal cells from wharton's jelly without enzymatic treatment," Stem Cells and Development, vol. 20, no. 3, pp. 547-557, 2011.

[59] M. Dominici, K. le Blanc, I. Mueller et al., "Minimal criteria for defining multipotent mesenchymal stromal cells. The International Society for Cellular Therapy position statement," Cytotherapy, vol. 8, no. 4, pp. 315-317, 2006. 
[60] M. T. Conconi, R. D. Liddo, M. Tommasini, C. Calore, and P. P. Parnigotto, "Phenotype and differentiation potential of stromal populations obtained from various zones of human umbilical cord: an overview," The Open Tissue Engineering and Regenerative Medicine Journal, vol. 4, pp. 6-20, 2011.

[61] R. Anzalone, M. L. Iacono, S. Corrao et al., "New emerging potentials for human wharton's jelly mesenchymal stem cells: immunological features and hepatocyte-like differentiative capacity," Stem Cells and Development, vol. 19, no. 4, pp. 423-438, 2010.

[62] K. I. Pappa and N. P. Anagnou, "Novel sources of fetal stem cells: where do they fit on the developmental continuum?" Regenerative Medicine, vol. 4, no. 3, pp. 423-433, 2009.

[63] U. Nekanti, L. Mohanty, P. Venugopal, S. Balasubramanian, S. Totey, and M. Ta, "Optimization and scale-up of Wharton's jellyderived mesenchymal stem cells for clinical applications," Stem Cell Research, vol. 5, no. 3, pp. 244-254, 2010.

[64] C. K. Tong, S. Vellasamy, B. C. Tan et al., "Generation of mesenchymal stem cell from human umbilical cord tissue using a combination enzymatic and mechanical disassociation method," Cell Biology International, vol. 35, no. 3, pp. 221-226, 2011.

[65] C.-Y. Fong, L.-L. Chak, A. Biswas et al., "Human Wharton's jelly stem cells have unique transcriptome profiles compared to human embryonic stem cells and other mesenchymal stem cells," Stem Cell Reviews and Reports, vol. 7, no. 1, pp. 1-16, 2011.

[66] R. S. Rachakatla, F. Marini, M. L. Weiss, M. Tamura, and D. Troyer, "Development of human umbilical cord matrix stem cell-based gene therapy for experimental lung tumors," Cancer Gene Therapy, vol. 14, no. 10, pp. 828-835, 2007.

[67] D.-W. Kim, M. Staples, K. Shinozuka, P. Pantcheva, S.-D. Kang, and C. V. Borlongan, "Wharton's jelly-derived mesenchymal stem cells: phenotypic characterization and optimizing their therapeutic potential for clinical applications," International Journal of Molecular Sciences, vol. 14, no. 6, pp. 11692-11712, 2013.

[68] K. Gauthaman, F. C. Yee, S. Cheyyatraivendran, A. Biswas, M. Choolani, and A. Bongso, "Human umbilical cord Wharton's jelly stem cell (hWJSC) extracts inhibit cancer cell growth in vitro," Journal of Cellular Biochemistry, vol. 113, no. 6, pp. 20272039, 2012.

[69] C.-Y. Fong, A. Biswas, A. Subramanian, A. Srinivasan, M. Choolani, and A. Bongso, "Human keloid cell characterization and inhibition of growth with human Wharton's jelly stem cell extracts," Journal of Cellular Biochemistry, vol. 115, no. 5, pp. 826-838, 2014.

[70] S. Wu, G.-Q. Ju, T. Du, Y.-J. Zhu, and G.-H. Liu, "Microvesicles derived from human umbilical cord Wharton's jelly mesenchymal stem cells attenuate bladder tumor cell growth in vitro and in vivo," PLoS ONE, vol. 8, no. 4, Article ID e61366, 2013.

[71] H. D. Lin, C. Y. Fong, A. Biswas, M. Choolani, and A. Bongso, "Human Wharton's jelly stem cells, its conditioned medium and cell-free lysate inhibit the growth of human lymphoma cells," Stem Cell Reviews and Reports, vol. 10, no. 4, pp. 573-586, 2014.

[72] T. Matsuzuka, R. S. Rachakatla, C. Doi et al., "Human umbilical cord matrix-derived stem cells expressing interferon- $\beta$ gene significantly attenuate bronchioloalveolar carcinoma xenografts in SCID mice," Lung Cancer, vol. 70, no. 1, pp. 28-36, 2010.

[73] C. Doi, D. K. Maurya, M. M. Pyle, D. Troyer, and M. Tamura, "Cytotherapy with naive rat umbilical cord matrix stem cells significantly attenuates growth of murine pancreatic cancer cells and increases survival in syngeneic mice," Cytotherapy, vol. 12, no. 3, pp. 408-417, 2010.
[74] R. Ayuzawa, C. Doi, R. S. Rachakatla et al., "Naïve human umbilical cord matrix derived stem cells significantly attenuate growth of human breast cancer cells in vitro and in vivo," Cancer Letters, vol. 280, no. 1, pp. 31-37, 2009.

[75] K. Gauthaman, C.-Y. Fong, S. Arularasu et al., "Human Wharton's jelly stem cell conditioned medium and cell-free lysate inhibit human osteosarcoma and mammary carcinoma cell growth in vitro and in xenograft mice," Journal of Cellular Biochemistry, vol. 114, no. 2, pp. 366-377, 2013.

[76] D. K. Maurya, C. Doi, A. Kawabata et al., "Therapy with unengineered naïve rat umbilical cord matrix stem cells markedly inhibits growth of murine lung adenocarcinoma," BMC Cancer, vol. 10, article 590, 2010.

[77] C. Ganta, D. Chiyo, R. Ayuzawa et al., "Rat umbilical cord stern cells completely abolish rat mammary carcinomas with no evidence of metastasis or recurrence 100 days post-tumor cell inoculation," Cancer Research, vol. 69, no. 5, pp. 1815-1820, 2009.

[78] I. Han, M. Yun, E.-O. Kim, B. Kim, M.-H. Jung, and S.-H. Kim, "Umbilical cord tissue-derived mesenchymal stem cells induce apoptosis in PC-3 prostate cancer cells through activation of JNK and downregulation of PI3K/AKT signaling," Stem Cell Research and Therapy, vol. 5, no. 2, article no. 54, 2014.

[79] X. Yang, Z. Li, Y. Ma et al., "Human umbilical cord mesenchymal stem cells promote carcinoma growth and lymph node metastasis when co-injected with esophageal carcinoma cells in nude mice," Cancer Cell International, vol. 14, no. 1, article 93, 2014.

[80] T. Du, G. Ju, S. Wu et al., "Microvesicles derived from human Wharton's jelly mesenchymal stem cells promote human renal cancer cell growth and aggressiveness through induction of hepatocyte growth factor," PLoS ONE, vol. 9, no. 5, Article ID e96836, 2014.

[81] M. Wang, Y. Yang, D. Yang et al., "The immunomodulatory activity of human umbilical cord blood-derived mesenchymal stem cells in vitro," Immunology, vol. 126, no. 2, pp. 220-232, 2009.

[82] C. Zhou, B. Yang, Y. Tian et al., "Immunomodulatory effect of human umbilical cord Wharton's jelly-derived mesenchymal stem cells on lymphocytes," Cellular Immunology, vol. 272, no. 1, pp. 33-38, 2011.

[83] M. L. Weiss, C. Anderson, S. Medicetty et al., "Immune properties of human umbilical cord Wharton's jelly-derived cells," Stem Cells, vol. 26, no. 11, pp. 2865-2874, 2008.

[84] S. Jyothi Prasanna and V. S. Jahnavi, "Wharton's jelly mesenchymal stem cells as off-the-shelf cellular therapeutics: a closer look into their regenerative and immunomodulatory properties," Open Tissue Engineering and Regenerative Medicine Journal, vol. 4, no. 1, pp. 28-38, 2011.

[85] S. Ma, N. Xie, W. Li, B. Yuan, Y. Shi, and Y. Wang, "Immunobiology of mesenchymal stem cells," Cell Death and Differentiation, vol. 21, no. 2, pp. 216-225, 2014.

[86] X.-P. Huang, Z. Sun, Y. Miyagi et al., "Differentiation of allogeneic mesenchymal stem cells induces immunogenicity and limits their long-term benefits for myocardial repair," Circulation, vol. 122, no. 23, pp. 2419-2429, 2010.

[87] N. Kotobuki, Y. Katsube, Y. Katou, M. Tadokoro, M. Hirose, and H. Ohgushi, "In vivo survival and osteogenic differentiation of allogeneic rat bone marrow mesenchymal stem cells (MSCs)," Cell Transplantation, vol. 17, no. 6, pp. 705-712, 2008.

[88] S. Liu, M. Yuan, K. Hou et al., "Immune characterization of mesenchymal stem cells in human umbilical cord Wharton's 
jelly and derived cartilage cells," Cellular Immunology, vol. 278, no. 1-2, pp. 35-44, 2012.

[89] S. Medicetty, A. R. Bledsoe, C. B. Fahrenholtz, D. Troyer, and M. L. Weiss, "Transplantation of pig stem cells into rat brain: proliferation during the first 8 weeks," Experimental Neurology, vol. 190, no. 1, pp. 32-41, 2004.

[90] J. Hu, X. Yu, Z. Wang et al., "Long term effects of the implantation of Wharton's jelly-derived mesenchymal stem cells from the umbilical cord for newly-onset type 1 diabetes mellitus," Endocrine Journal, vol. 60, no. 3, pp. 347-357, 2013.

[91] X. Liu, P. Zheng, X. Wang et al., "A preliminary evaluation of efficacy and safety of Wharton's jelly mesenchymal stem cell transplantation in patients with type 2 diabetes mellitus," Stem Cell Research and Therapy, vol. 5, no. 2, article no. 57, 2014.

[92] Q. Chen, K. Liu, A. R. Robinson et al., "DNA damage drives accelerated bone aging via an NF- $\kappa \mathrm{B}$-dependent mechanism," Journal of Bone and Mineral Research, vol. 28, no. 5, pp. 12141228, 2013.

[93] G. Siegel, T. Kluba, U. Hermanutz-Klein, K. Bieback, H. Northoff, and R. Schäfer, "Phenotype, donor age and gender affect function of human bone marrow-derived mesenchymal stromal cells," BMC Medicine, vol. 11, article 146, 2013.

[94] D. Wang, J. Li, Y. Zhang et al., "Umbilical cord mesenchymal stem cell transplantation in active and refractory systemic lupus erythematosus: a multicenter clinical study," Arthritis Research and Therapy, vol. 16, no. 2, article R79, 2014.

[95] Y. Wang, F. Chen, B. Gu, G. Chen, H. Chang, and D. Wu, "Mesenchymal stromal cells as an adjuvant treatment for severe late-onset hemorrhagic cystitis after allogeneic hematopoietic stem cell transplantation," Acta Haematologica, vol. 133, no. 1, pp. 72-77, 2015. 


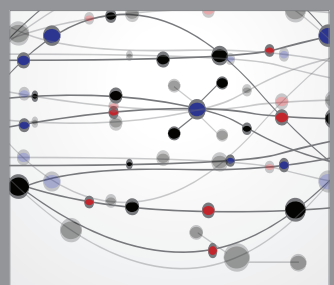

The Scientific World Journal
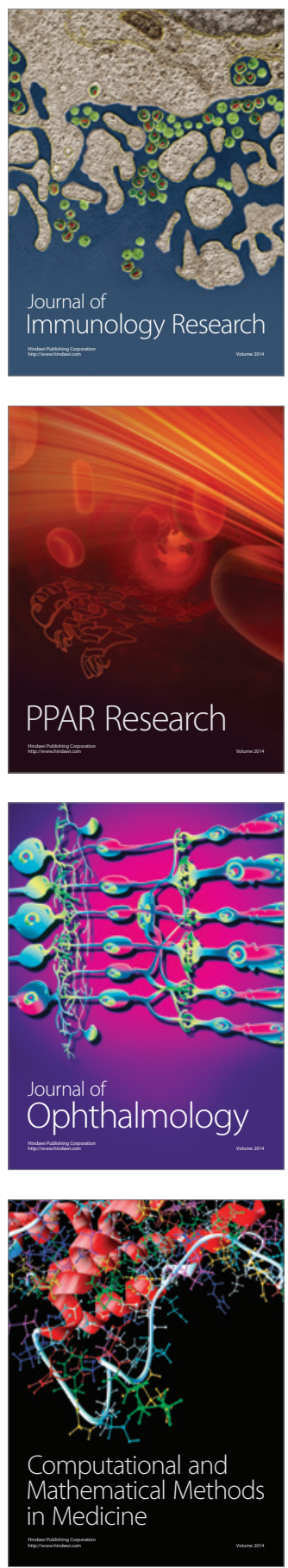

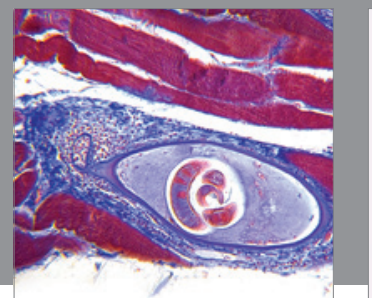

Gastroenterology

Research and Practice
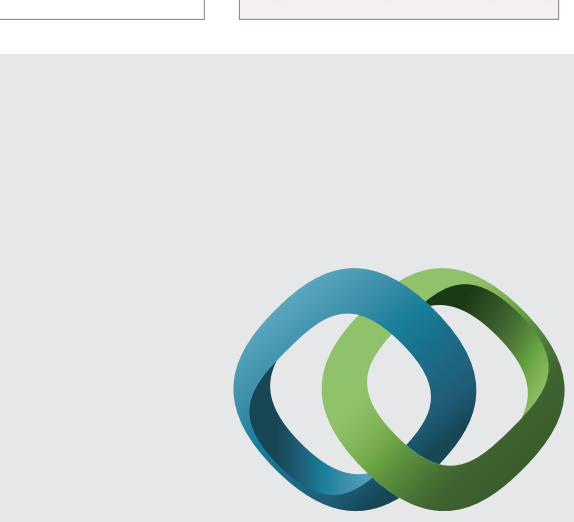

\section{Hindawi}

Submit your manuscripts at

http://www.hindawi.com
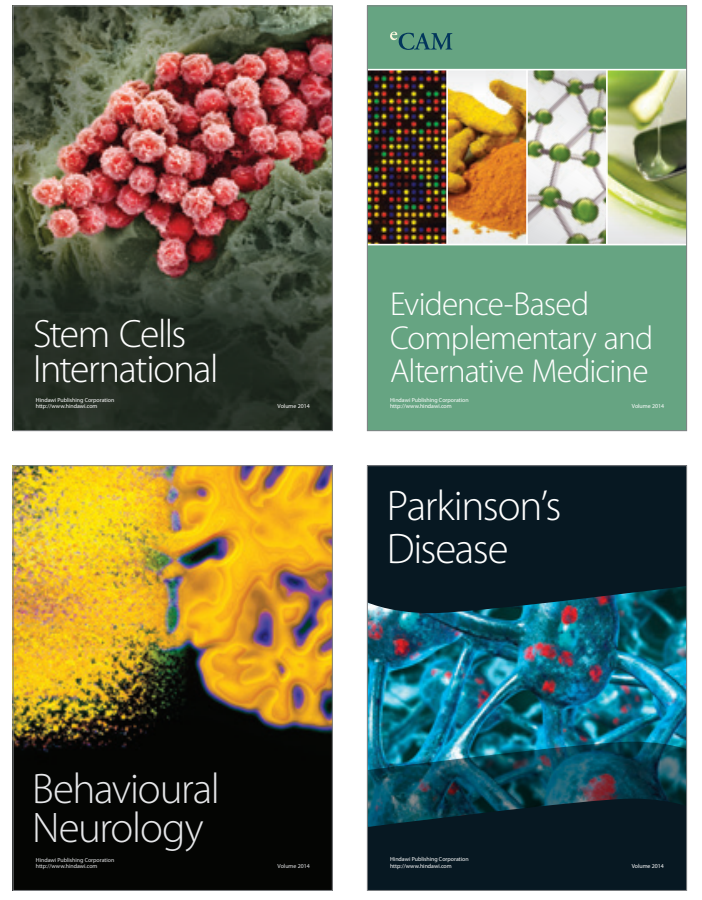
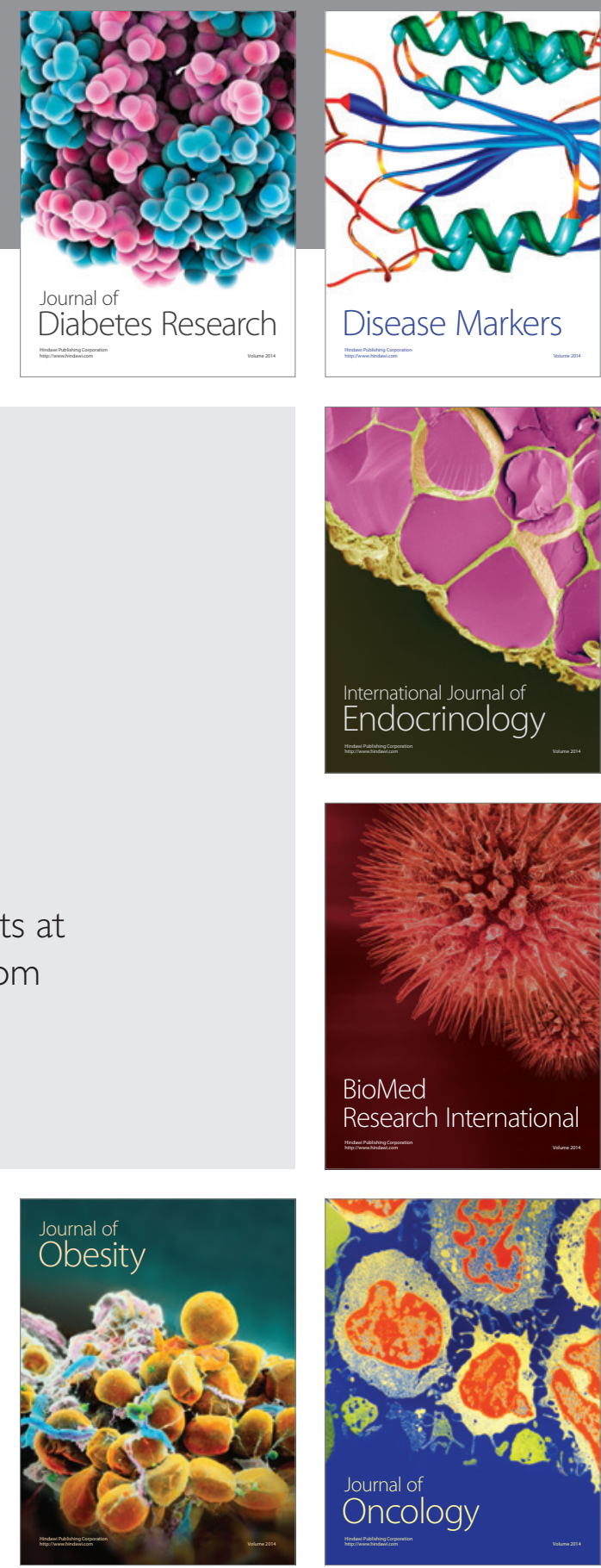

Disease Markers
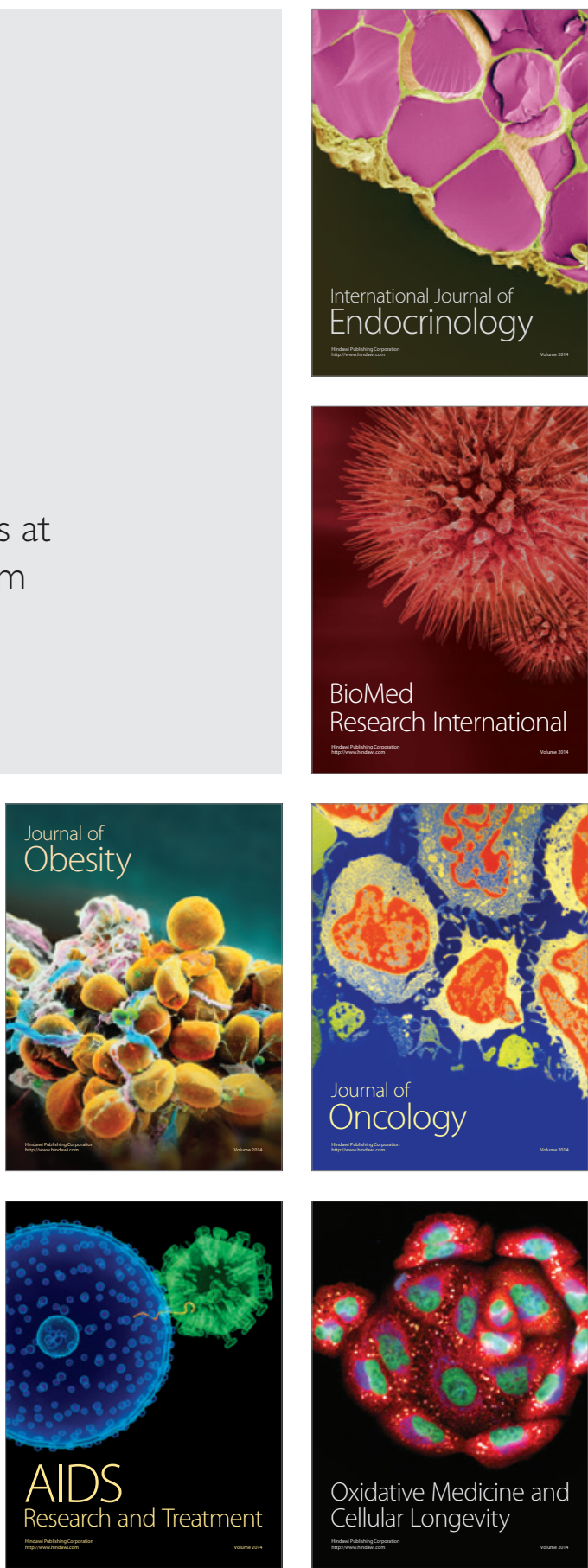